\title{
Direct visualization of bimodal-propagation-induced spatial self-imaging
}

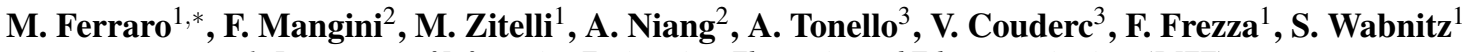 \\ 1. Department of Information Engineering, Electronics and Telecommunications (DIET), \\ Sapienza University of Rome, Via Eudossiana 18, 00184 Rome, Italy \\ 2. Department of Information Engineering (DII), University of Brescia, Via Branze 38, 25123 Brescia, Italy \\ 3. Université de Limoges, XLIM, UMR CNRS 7252, 123 Avenue A. Thomas, 87060 Limoges, France \\ mario.ferraro@uniromal.it
}

Spatial self-imaging (SSI) consists of the periodic replication of the transverse profile of an optical beam along its propagation. It can be achieved in guided wave systems when all excited modes interfere in phase [1]. Remarkable is the case of graded-index multimode fibers, where the modes propagation constants are equally spaced, and the interference condition becomes trivial, giving rise to a periodic breathing of the field effective area [2]. In the singlemode propagation regime, SSI is by definition impossible. However, bimodal propagation can be attained in a nominal singlemode fiber (SMF-28), when operating in proximity of its cut-off wavelength. The latter imposes the condition for which only the fundamental mode is guided, so that higher-order modes (HOM) vanish over a certain damping distance (typically of about a few centimeters, depending on the wavelength). Therefore, effects associated to the presence of HOM can be present, over propagation lengths shorter than the damping distance.

Here we report the direct experimental visualization of SSI in a bimodal silica SMF-28, by exploiting the material defect photoluminescence which occurs the first centimeters of propagation. Visible luminescence is excited via the multiphoton absorption of MW peak power femtosecond laser pulses [3].

a)

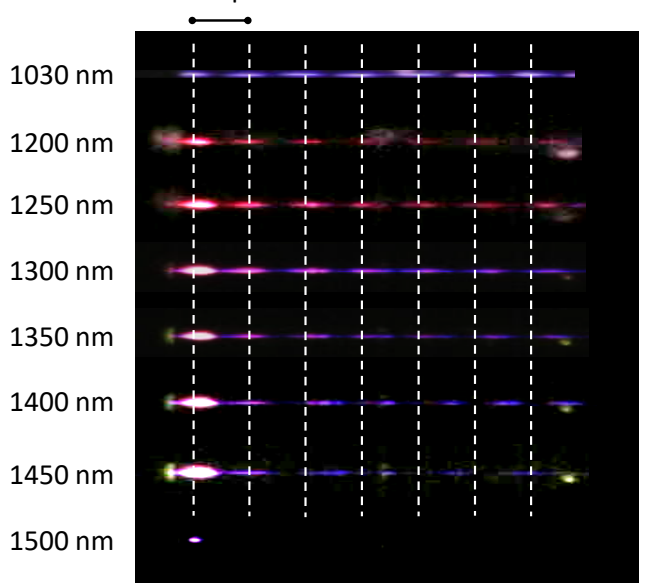

b)

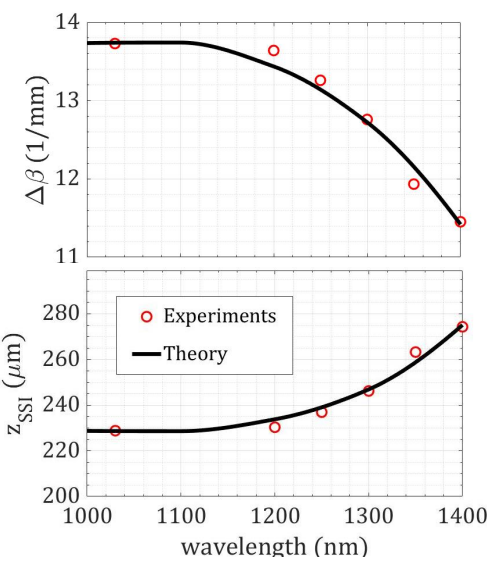

Fig. 1. (a) True color picture of the fiber photoluminescence when varying the input wavelength; (b) Comparison between theoretical and experimental values of the spatial self-imaging period and propagation constant difference between the $L P_{01}$ and $L P_{11}$ modes.

In Fig.1a we show how the SSI varies with the input wavelength, and it eventually vanishes above $1450 \mathrm{~nm}$ when crossing the cut-off wavelength. The theoretical SSI period $\left(z_{S S I}\right)$ is determined by the constructive interference condition, that in a bimodal regime trivially reads:

$$
\Delta \beta \cdot z_{S S I}=2 \pi
$$

where $\Delta \beta$ is the propagation constant difference between the first excited and the fundamental mode $\left(L P_{01}\right.$ and $L P_{11}$, respectively). In Fig. $1 \mathrm{~b}$ we show that a good agreement is found between theoretical and experimental values of $z_{S S I}$ and $\Delta \beta$, respectively. Our results provide a new method to estimate the mode propagation constants of fibers, which is of utmost importance for telecom applications. Furthermore, the SSI period estimation is a previously undisclosed way to empirically determine the value of the fiber cut-off, independently of fiber length. We acknowledge support from EU Horizon2020 ERC Grants No. 874596 and No. 740355.

[1] S. Allison and G. Gillies, "Observations of and applications for self-imaging in optical fibers," Appl. optics 33, 1802-1805 (1994).

[2] T. Hansson et al., "Nonlinear beam self-imaging and self-focusing dynamics in a GRIN multimode optical fiber: theory and experiments," Opt. Express 28, 24005-24021 (2020).

[3] F. Mangini et al., "Multiphoton-absorption-excited up-conversion luminescence inoptical fibers," Phys. Rev. Appl. 14, 054063 (2020). 